\title{
Entwicklung einer neuen Methode zur Untersuchung von Strahlenschäden bei kleinen Dosen
}

\author{
Heimgartner, E., Reist, H.W., Kelemen, A., Kohler, M.
}

Institut für Medizinische Radiobiologie der Universität Zürich und des Paul Scherrer Instituts, CH-5232 Villigen PSI, Schweiz

\section{EINLEITUNG:}

Bei kleinen Strahlendosen (z.B. Radon oder berufliche Exposition) ist die Wahrscheinlichkeit, dass ein Zellkern von mehr als einem geladenen Teilchen getroffen wird, vernachlässigbar klein. Es wird allgemein angenommen, dass Krebs aus einer einzigen überlebenden, aber transformierten Zelle entstehen kann. Für Risikoabschätzungen im Strahlenschutz ist es deshalb wichtig, die Wirkung einzelner Teilchendurchgänge durch den Zellkern individueller Zellen auf Überleben, sowie spezifische Chromosomenaberrationen (Translokationen, Deletionen) zu untersuchen.

In bisherigen Arbeiten wurde abgeschätzt, dass im Mittel zwei bis sechs Durchgänge von $\alpha$-Teilchen mit hohem LET genügen, um die Zellen zu inaktivieren [1].

Nelson et al. [2] beobachteten Mikrokernbildung in CHO-K1-Zellen nach einem Durchgang eines einzigen 3.5 MeV $\alpha$-Teilchens.

Im vorliegenden Beitrag wird eine neue Methode vorgestellt, mit der (i) eine grosse Zahl von Einzelzellen oder kleine Zellklone mit geladenen Teilchen bestrahlt und (ii) durch Messen der Spurpositionen die Intersektionslängen der Teilchen durch den Zellkem, und daraus die Energieabgabe bestimmt werden kann. Mit dieser Methode wird in einer ersten Studie das Koloniewachstum von V79 Hamsterzellen als Funktion der Energieabgabe von $9.6 \mathrm{MeV} \alpha$-Teilchen untersucht.

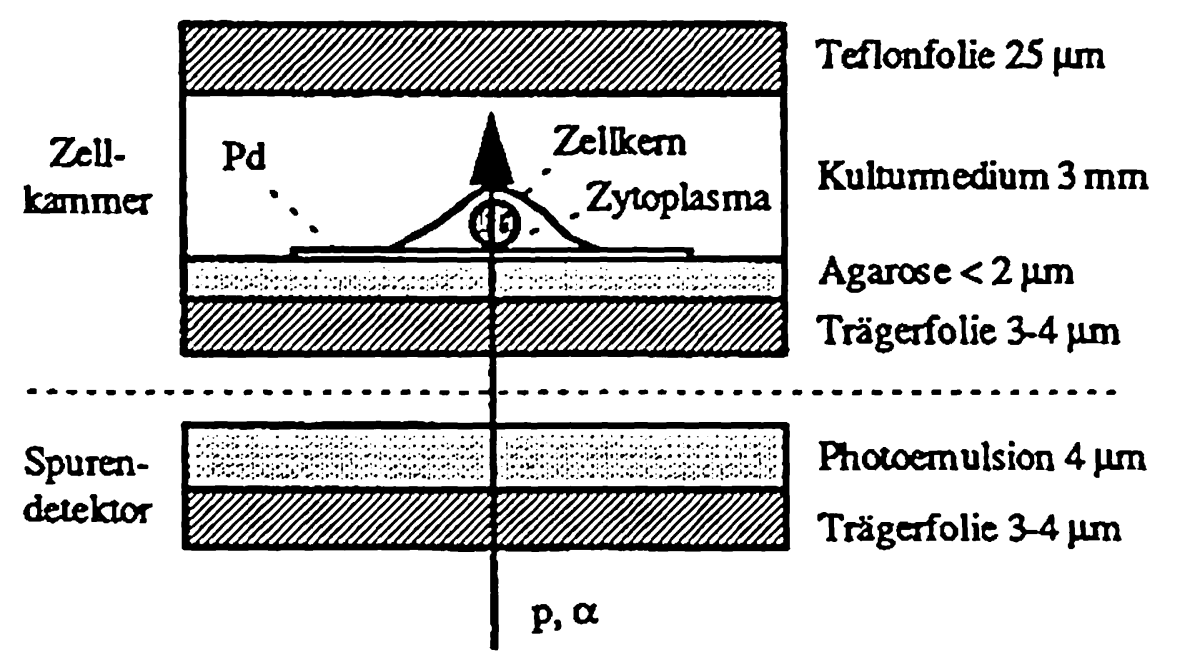

Figur 1: Experimenteller Aufbau bestehend aus der Zellkammer mit den Zellen auf den Pd-Inseln und dem Spurendetektor

\section{METHODE:}

Der experimentelle Aufbau besteht aus einer speziell entwickelten Zellkammer mit einem Spurendetektor (Figur 1). Die Zellen wachsen auf über 1000 regelmässig angeordneten Pd-Inseln, die auf einer Agarose-Schicht aufgedampft sind. Damit kann das Koloniewachstum von bestrahlten Einzelzellen untersucht werden. Die Bestrahlungskammer und die Strahlführung sind in [3] und [4] bereits beschrieben.

Der Spurendetektor besteht aus einer dünnen Schicht Photoemulsion K5 (llford), die auf einer 3-4 $\mu \mathrm{m}$ dicken Folie aufgetragen ist. Bei der photographischen Entwicklung der Spuren dehnt sich die Folie bis zu $5 \mu \mathrm{m}$ radial aus. Die entstehenden Verzerrungen werden korrigiert. Polypropylenfolien lieferten die besten Ergebnisse bezüglich Verzerrungen und optischer Qualität. Mittels konfokaler Mikroskopie (CLSM) kann der Untergrund bei der Spurenidentifikation weitgehend unterdrückt werden (Figur 2).

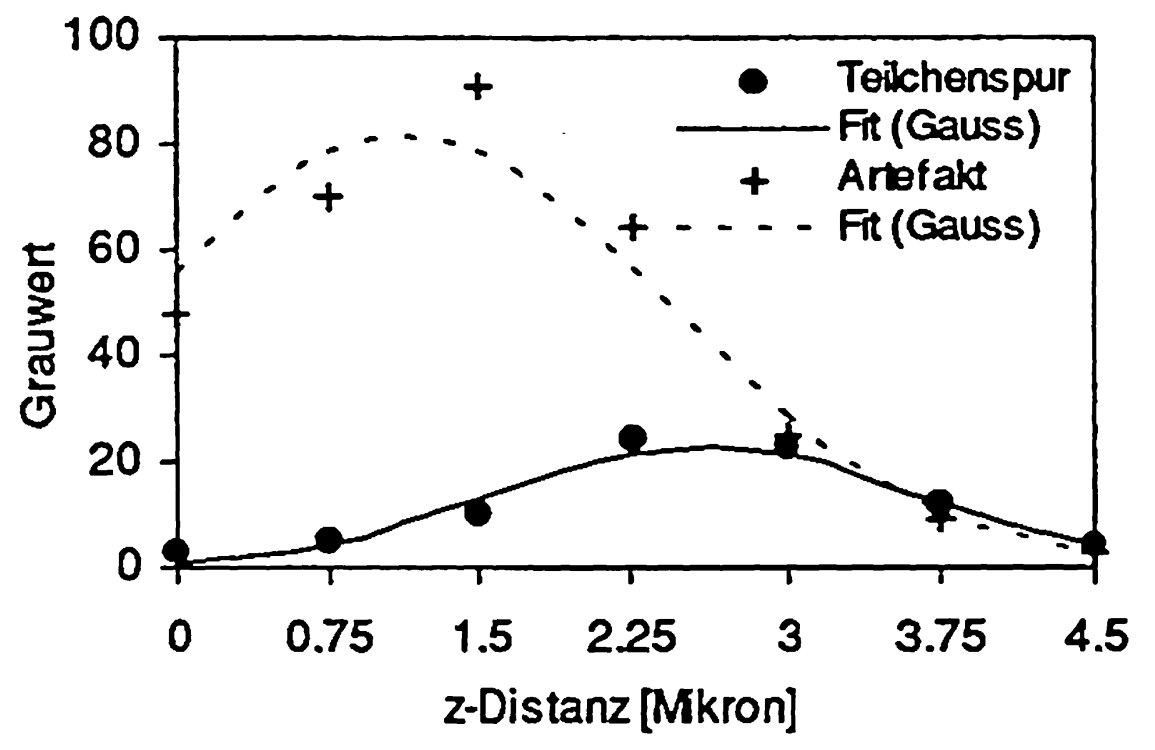

Figur 2: Identifikation der Teilchenspuren durch Analyse von 7 konfokalen CLSM-Schnitten (ReflexionsMode). Die Lage des Maximums und die Halbwertsbreite dienen zur Trennung der Spuren in der Emulsionsschicht von den Artefakten in der Trägerfolie.

Der Ort eines Teilchendurchgangs durch den Zellkem wird durch digitale Überlagerung des PhasenkontrastBildes der Zelle mit dem Spurenbild gemessen. Die Korrektur der Spurpositionen wird mit. Hilfe der Relativpositionen von kreisförmigen Aperturen auf der Folie der Zellen und ihren lichtprojizierten Bildem auf 
dem Spurendetektor berechnet. Die Genauigkeit der Ortsbestimmung wurde mit zusătzlichen, gleichmăssig verteilten Aperturen an Stelle von Zellpositionen ermittelt (Figuren 3 und 4).

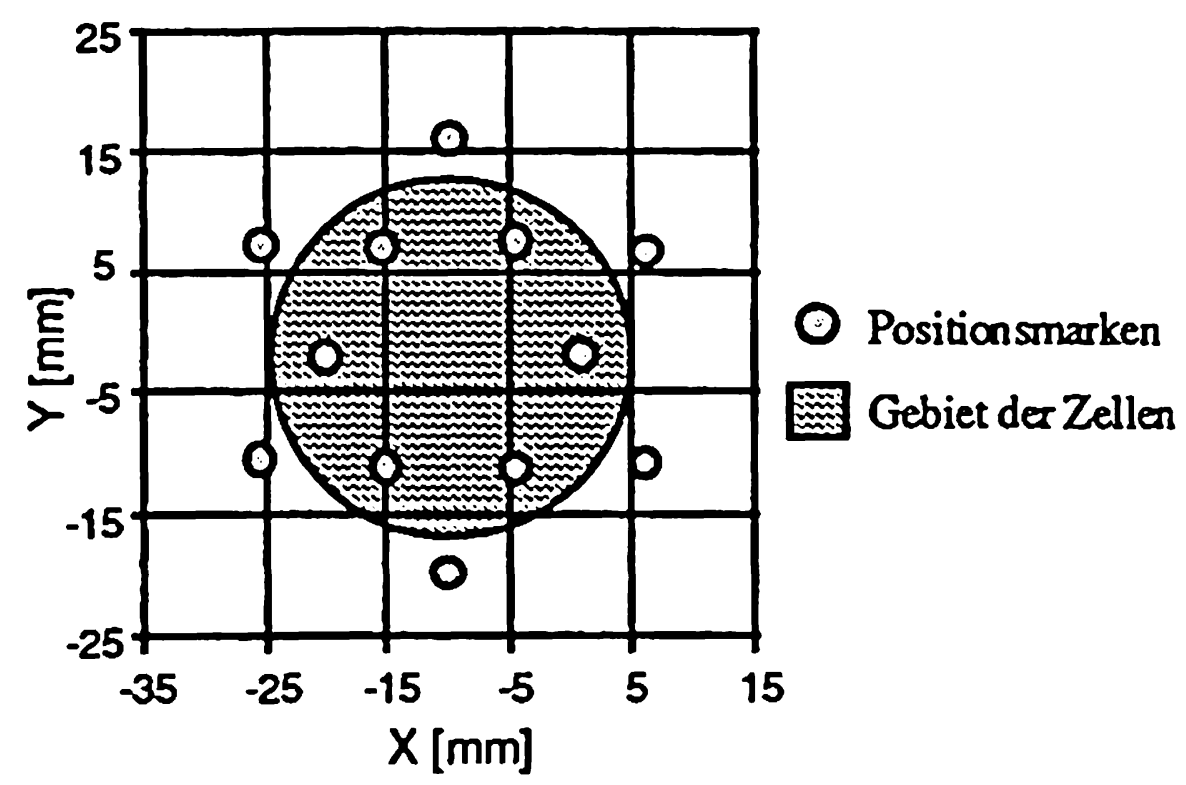

Figur 3: Die Korrelation von Zell- und Spurpositionen wird erreicht durch Ausmessen von Positionsmarken auf der Trägerfolie der Zellen relativ zu den Positionen ihrer lichtprojizierten Bilder auf dem Spurendetektor.

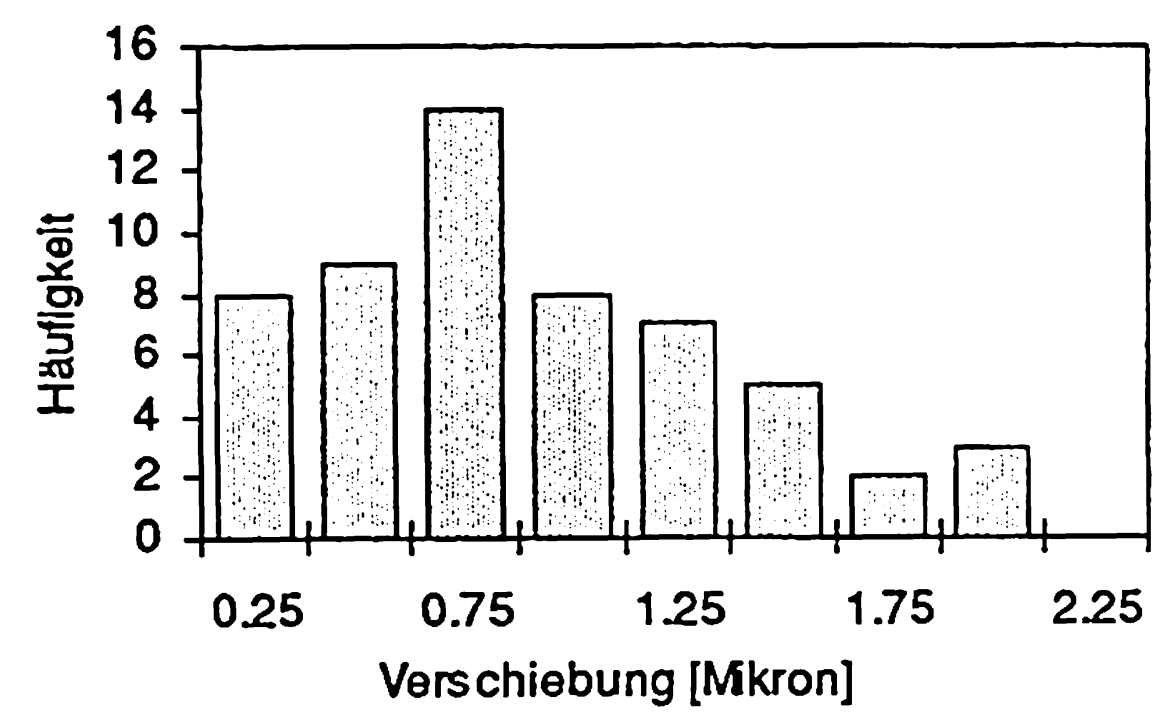

Figur 4: Die gemessene Korrelationsgenauigkeit beträgt für eine Polypropylenfolie von 4 um Dicke $(0.9 \pm 0.5)$ $\mu \mathrm{m}$. Sie wurde bestimmt durch das Anbringen zusätzlicher Positionsmarken an Stelle der Zellenpositionen.

Ein statistisches Modell der Oberflächenkontur der Zellkerne wird durch Segmentation von CLSM-Bildem bestimmt [5]. Aus dem lateralen Abstand der Teilchenspur von der Mitte des Zellkerns wird die Intersektionslänge und daraus die abgegebene Energie berechnet.

\section{RESULTATE:}

Aus der in Figur 4 dargestellten Häufigkeitsverteilung der Verschiebung zwischen den Referenzaperturen und ihren lichtprojizierten Bildern ergab sich eine Korrelationsgenauigkeit von $(0.9 \pm 0.5) \mu \mathrm{m}$.

In Figur 5 sind erste Daten zum Koloniewachsum bestrahlter V79 Hamsterzellen in Abhängigkeit der Energieabgabe im Zellkern dargestellt. Die Energie der $\alpha$-Teilchen betrug $9.6 \mathrm{MeV}$, der LET $57.3 \mathrm{keV} / \mu \mathrm{m}$. Die

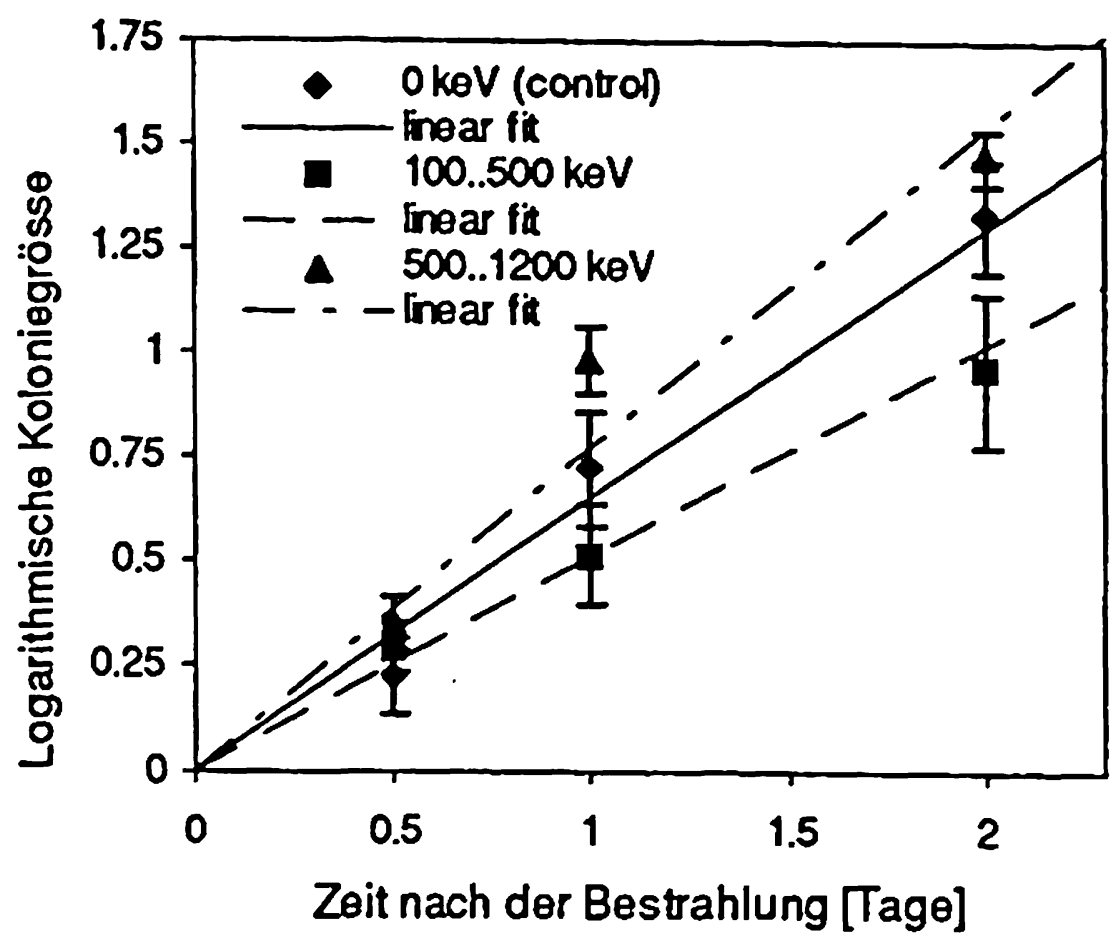

Figur 5: Koloniewachstum von V79 Hamsterzellen fur verschiedene Energieabgaben im Zellkern der bestrahlten Zelle. Als vorläufige Parameter der Wachstumsgeschwindigkeit wurden 0.65/d (Kontrolle), $0.51 / d\left(E_{\text {trensfer }}=100.500 \mathrm{keV}\right)$ und $0.77 / d\left(E_{\text {transfar }}=\right.$ $500 . .1200 \mathrm{keV})$ geschätzt.

V79-Kerne waren annhähernd kugelförmig mit einem mittleren Durchmesser von $9 \mu \mathrm{m}$. Der berechnete Energietransfer liegt im Bereich von 100 bis $1200 \mathrm{keV}$, entsprechend einer individuellen Dosis von 5 bis $60 \mathrm{cGy}$.

\section{DISKUSSION:}

Die entwickelte Methode erlaubt die Identifikation der getroffenen Zellen und die Bestimmung der übertragenen Energie (individuelle Dosis). Mit der erreichten Korrelationsgenauigkeit lässt sich die biologische Wirkung einzelner Teilchendurchgänge untersuchen. Die Pd-Insel-Technik gestattet zusammen mit der Ortsbestimmung des Teilchendurchgangs die Untersuchung des Koloniewachstums und die Bestimmung der Wachstumsgeschwindigkeit des Klons als Funktion der Energieabgabe an die bestrahlte Einzelzelle.

\section{VERDANKUNGEN:}

Wir möchten Roger Leemann für hilfreiche Diskussion und allgemeine Hilfe, Lucie Hofmann für die wertvolle Mitarbeit bei der Vorbereitung und Durchführung der Experimente, Dr. Jiri Stepanek für die genaue Berechnung der LET-Werte und Takashi Suda für die technische Unterstützung herzlich danken.

\section{LITERATUR:}

[1] M.R. Raju et al.; Radiat. Res. 128, 204 (1991)

[2] J.M. Nelson et al.; Radiat. Res. 145, 568 (1996)

[3] H.W. Reist et al.; Radiat. Prot. Dosimetry 61, 221 (1995)

[4] H.W. Reist et al.; SGSMP-SGBT Tagungsberichte, Villigen, Schweiz, 211 (1993)

[5] A. Kelemen et al.; Visualization in Biomedical Computing, Hamburg, Sept. 22-25 (1996) 